EPJ Web of Conferences 64, 08001 (2014)

DOI: $10.1051 /$ epjconf/ 20146408001

(C) Owned by the authors, published by EDP Sciences, 2014

\title{
Observational clues to the physics at the magnetosphere in young stellar objects
}

\author{
Silvia H. P. Alencar ${ }^{1, a}$ \\ ${ }^{1}$ Departamento de Física - ICEx - UFMG, Av. Antônio Carlos, 6627, 30270-901, Belo Horizonte, MG, Brazil
}

\begin{abstract}
Classical T Tauri stars offer an excellent laboratory to study the accretion process and star-disk interaction, which define the transfer of mass and angular momentum in the system. The interaction takes place very close to the star, in a region of about a few stellar radii, and it is therefore challenging to study in detail the physics of the magnetosphere in young stellar objects. We will discuss, through observational examples, how our description of a static and axisymmetric stellar magnetosphere from the early 90s has evolved to a very dynamical multipolar region that takes into account non-steady accretion and time-dependent star-disk interactions.
\end{abstract}

\section{Introduction}

Classical T Tauri stars (CTTSs) are young ( 1 Myr) low-mass $\left(\leq 2 \mathrm{M}_{\odot}\right)$ stars that show evidence of accretion from a circumstellar disk. Their observed characteristics have been successfully described over the years by magnetospheric accretion models [12, 28, 30, 32, 33, 36, 41]. In theses models, the stellar magnetic field is strong enough to truncate the circumstellar disk at a few stellar radii from the star, where the magnetic stress equals the stress of the accreting matter, forming a magnetospheric cavity. The accreting material reaches the inner disk region and, if it is sufficiently ionized, its motion will be controlled by the magnetic field. Part of it will be ejected in a wind and part will accrete onto the star, following closed magnetic field lines and forming accretion columns. The accreting material reaches the star at near free-fall velocities, forming a strong shock region and a hot spot.

The rich environment of a CTTS offers many observational diagnostics to analyse the accretion process. The intensity and topology of the stellar surface magnetic field can be obtained through the broadening of Zeeman sensitive lines [29, 43] and Zeeman Doppler Imaging (ZDI) [16, 17]. Ultraviolet (UV) and optical excess, that veils the photospheric lines, are produced at the hot spot $[5,26]$. Soft $\mathrm{X}$-ray emission comes from the shock region $[25,40]$ that is also the origin of narrow emission components of lines such as HeI (5876 ̊) [6]. In high mass-accretion stars, a strong stellar wind seems to be present, as inferred by P Cygni profiles of the HeI (10830 ̊) line [21]. In the accretion column, as the accreting gas is heated, mostly by adiabatic compression, strong and wide emission lines, such as hydrogen lines, are produced [36]. Photons from the hot spot are absorbed by the accretion column and show up as redshifted absorption components in systems seen at high inclination with respect to

ae-mail: silvia@fisica.ufmg.br

This is an Open Access article distributed under the terms of the Creative Commons Attribution License 2.0, which permits unrestricted use, distribution, and reproduction in any medium, provided the original work is properly cited. 
our line of sight [20]. The observed infrared excess comes from a combination of viscous dissipation and reprocessing of stellar and accretion radiation by the inner disk, while blueshifted absorption components in emission lines and forbidden emission lines are produced in the wind [27].

In recent years it became clear that the physical processes at the magnetosphere are intrinsically variable. According to observations, the stellar magnetic field varies in strength and topology in a timescale of years [18, 19], the star-disk interaction varies in a timescale of days/weeks [9], the accretion process may vary in several timescales, from hours (stochastic accretion, [42]) to month/years (EXOrs and FU Ori outbursts $[4,44]$ ). Added to all this there is also rotational modulation with typical periods of a few days $[1,7]$. At the same time that observations pointed to time variable accretion features, magnetospheric accretion models evolved from steady-state axisymmetric stellar dipoles to 3D magneto-hydrodynamical (MHD) simulations of star-disk interaction with inclined multipolar stellar magnetic fields [37, 39]. The observational and theoretical results indicate that a lot can be learned about the accretion process and the circumstellar environment of CTTSs in the time domain.

There are now a few CTTS systems, such as V2129 Oph and AA Tau that have been followed during several rotational cycles in different seasons in a broad wavelength range, using different observational techniques (photometry, spectroscopy, spectropolarimetry) simultaneously. The observed data has also been extensively modeled by several groups. These systems produced excelent data to explore the star-disk interaction and the accretion process variability and will be reviewed in Sects. 2 and 3.

It is however very difficult and telescope time consuming to obtain detailed observing campaigns of a large number of CTTSs, covering many wavelength ranges over several rotational cycles. We must therefore ask ourselves if we can extrapolate the results we find for the few well studied CTTSs to the entire CTTS class. The recent multiwavelength campaign of observations of the young stellar cluster NGC 2264 [13] can give clues on that, as shown in Sect. 4 (see also the contributions of Nathalia Fonseca and Ann Marie Cody in this volume).

\section{V2129 Oph}

V2129 Oph is a K5 CTTS located in the $\rho$ Oph star forming region and seen at moderate inclination $\left(i \sim 60^{\circ}\right)$ with respect to our line of sight. A multiwavelength observing campaign of this system was organized in 2009, which included simultaneous optical and near-IR photometry, high resolution spectroscopy and spectropolarimetry, and Chandra X-ray observations [2, 3, 18].

The stellar magnetic field at the surface of the star was obtained in two different epochs (2005 and 2009) and was found to be composed of a dipole and an octupole tilted by about $20^{\circ}$ with respect to the rotation axis. The intensities of each multipole varied between the two epochs, pointing to a non-fossil origin for the stellar magnetic field [18].

The proposed magnetic field strength and topology obtained was tested with data available from the campaign. The veiling of photospheric lines comes from the hot spot continuum emission, and its modulation, as the system rotates, is related to the hot spot characteristics. The HeI (5876 $\mathrm{A}$ ) line also comes from the shock region and its radial velocity variability is due to the rotation of the hot spot with respect to our line of sight. The prediction of hot spot filling factor and location from the ZDI results was confirmed through the modeling of both veiling variability and HeI (5876 $⿱$ A) line radial velocity variations [2].

The photometric variability of V2129 Oph in BVRI could only be modeled by a major cold spot at the stellar surface, that is almost coincident in phase with the inferred hot spot, as also predicted by the ZDI results. Due to the presence of a major cold spot, the photospheric lines get distorted as the cold spot comes in and out of view and their radial velocity is therefore related to the cold 


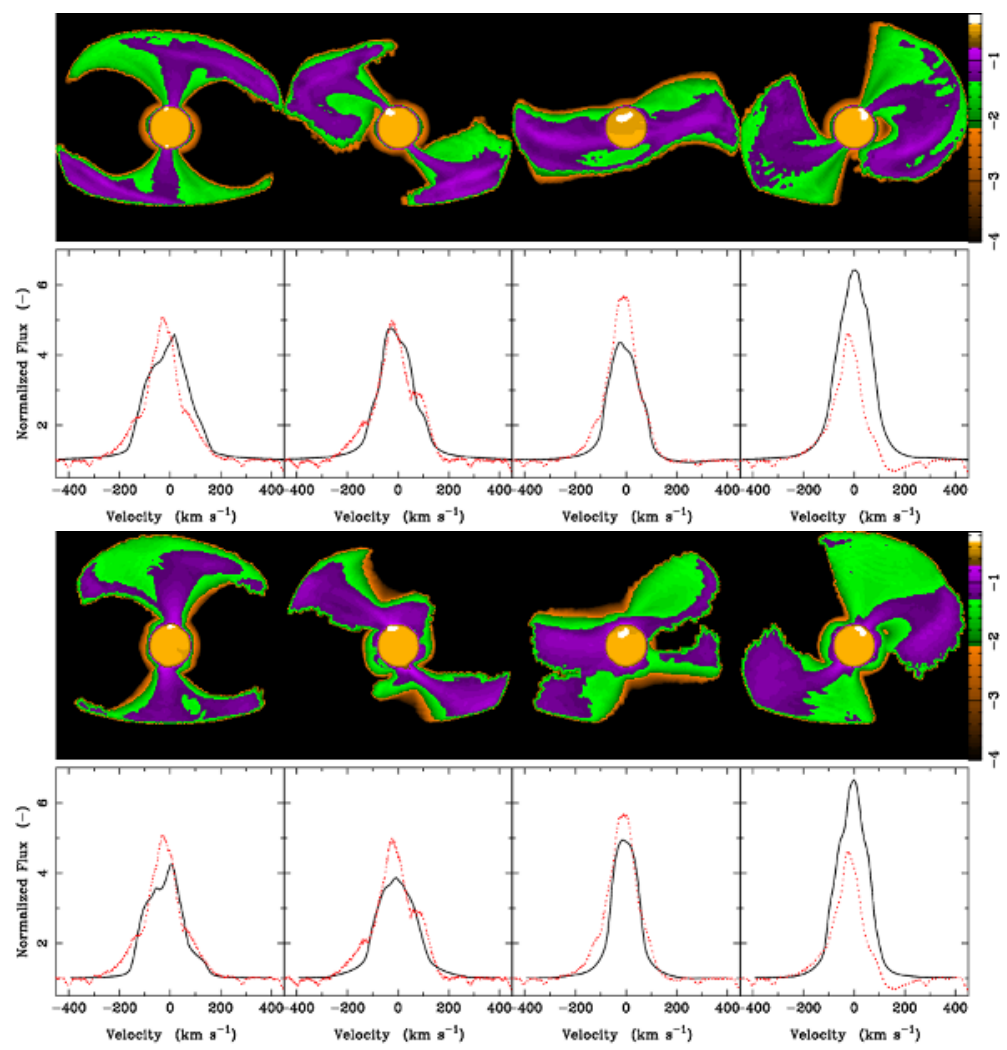

Figure 1. $\mathrm{H} \alpha$ model intensity maps (top panels) and the corresponding theoretical profiles (bottom panels, solid lines) computed from the 3D MHD simulation with a dipole-only (left figure) and a dipole+octupole (right figure) magnetic field at four rotational phases. The profiles observed with the HARPS spectrograph (dotted lines) are shown for comparison. Figure taken from [2].

spot characteristics. The modeling of the photospheric radial velocity variations further confirmed the results obtained with the light curve analysis [2].

We were subsequently very confident that the proposed magnetic intensity and topology at the stellar surface was close to reality. We decided to try to reproduce the observed $\mathrm{H} \alpha$ and $\mathrm{H} \beta$ line variability with radiative transfer calculations [32] based on the 3D MHD structure of the proposed magnetic configuration [39]. These lines were expected to come primarily from the accretion columns and were not used in the calculation of the surface magnetic field. Two different models were tested, a pure dipole and the dipole+octupole magnetic field proposed by [18].

The 3D simulations show that in both the dipole and the dipole+octupole cases, the dipole component strongly dominates the field at large distances from the star and defines the truncation radius of the disk at about the same location. Near the stellar surface, the octupole component dominates the flow and redirects the accretion funnel to higher latitudes, and closer to the magnetic pole, than in the case of a pure dipole. In the dipole+octupole case, the cold and hot spots coincide better than in the pure dipole models, as observed. 
Given all the approximations involved, the results of the line profile calculations were extremely interesting (see Fig. 1). The theoretical line profiles closely resembled the observed ones and most of the observed line variability was also present in the calculated line profiles in the two magnetic field configurations. Some discrepancies remain, as can be seen in Fig. 1, but overall the magnetic field configuration obtained from ZDI reproduces quite well the $\mathrm{H} \alpha$ and $\mathrm{H} \beta$ emission line profiles of V2129 Oph.

\section{AA Tau}

AA Tau is a K7 CTTS seen at high inclination $\left(i \sim 75^{\circ}\right)$ with respect to our line of sight, which was the focus of many observing campaigns in the last decades [8-10]. It shows periodical ( $p \sim 8.2$ days) eclipses of the photosphere that change in width and depth from cycle to cycle (Fig. 2 top-left). They occur without much color variation, and the linear polarization increases as the system fades [35]. The eclipses are due to periodical occultation of the photosphere by an optically thick, magneticallywarped inner disk region [14]. This warp is thought to be created by the interaction of the inner disk region with the stellar magnetic field that is inclined with respect to the stellar rotation axis. The inclined magnetic field creates a major funnel flow in each disk hemisphere that interacts with the inner disk, favoring accretion at certain orbital phases. This scenario explains the periodical eclipses as due to the inner disk warp at the base of the major accretion funnel. The period of the eclipses correspond to the stellar rotation period (measured from radial velocity variations of the photopheric absorption lines) and indicates that the disk is truncated close to the co-rotation radius. The presence of a major accretion funnel is also confirmed by the redshifted absorptions close to the photometric minima present in the $\mathrm{H} \beta$ line, when the funnel is facing the observer (Fig. 2 middle-left). The veiling increases as the system fades, indicating that during the minima, the hot spot at the base of the accretion funnel, is also facing us (Fig. 2 bottom-left). The proposed scenario for AA Tau is in agreement with 3D MHD simulations that show the development of a major accretion flow, when the rotation and magnetic axis are misaligned [37].

This scenario is also consistent with optical and simultaneous UV and X-ray observations of AA Tau made by [24] with XMM-Newton. The UV flux was shown to be periodic at the stellar rotation period ( 8.2 days) but also at a much small timescale of $0.87 \mathrm{~d}$ that was tentatively attributed to nonsteady accretion. They observed a delay between the optical and UV eclipses that indicated a trailing accretion flow. There was no correlation between the UV and X-ray fluxes and no eclipses in X-rays, showing that the X-ray emitting regions are located at high latitudes, above the high-density warp.

Numerical simulations have also shown a dynamical interaction between the inner disk and the stellar magnetosphere. According to many simulations, differential rotation along the field lines, which are anchored to the disk at several radii and not only at co-rotation, leads to field line expansion, opening and reconnection, restoring the initial configuration, as the system rotates $[23,34,38,45,46]$. The timescale for this to occur is predicted to be of a few rotation periods but it is determined by the diffusion of the magnetic field through the inner disk regions, which is a poorly known parameter. The $\mathrm{H} \alpha$ line of AA Tau presents an emission component thought to be formed in the accretion funnel, and two absorption components, a blueshifted one (always present) from a wind and a redshifted one (present at certain phases) that comes from hot spot photons absorbed by the accretion funnel. It was shown that the opening of the field lines could be measured by the variation of the projected radial velocity of the redshifted absorption component of $\mathrm{H} \alpha$ [9]. This was the observational confirmation of the numerical models prediction.

The magnetic field topology of AA Tau was measured twice (2007 and 2009) with spectropolarimetry [17] and was found to vary but not by much between the two epochs. It is composed of a 

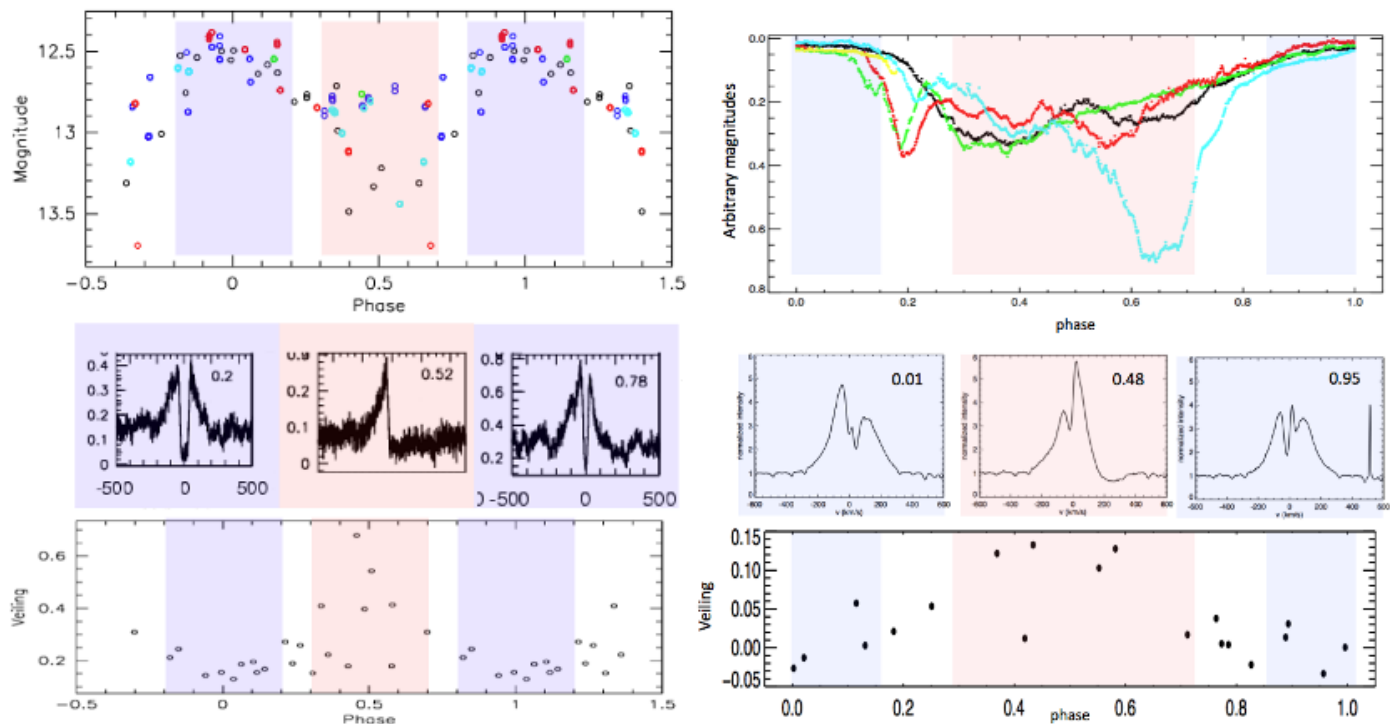

Figure 2. Left: AA Tau. Right: V422 Mon, an AA Tau-like star identified in the NGC 2264 cluster (see Sect. 4). Top panels: light curves folded in phase with the rotational period of each star ( 8.22 days for AA Tau and 8.6 days for V422 Mon). The light curves present well defined maxima interrupted by minima that vary in width and depth due to an inner disk warp. Middle panels: Balmer emission lines at phases outside the eclipses (blue background) and inside the eclipses (rose background), where we clearly see the redshifted absorptions when the main funnel flow is in our line of sight. Bottom panels: veiling at different rotational phases. The maximum veiling is observed close to phase 0.5 , when the hot spot is facing us.

simple axisymmetric field inclined with respect to the rotation axis of the star and presents a strong $\mathrm{kG}$ dipole and a weaker octupole component.

The eclipses seen in the AA Tau light curve were present for more than 20 years of photometric follow-up [10]. In a timescale of a weeks, they changed from cycle to cycle, sometimes almost disappearing for one or two rotational cycles (when accretion decreased), but beeing restablished with about the same shape. The shape of the obscuring screen, however, varies significantly over the years, suggesting that the star-disk interaction also varied significantly in that timescale. This could be due to changes in the magnetospheric configuration or in the acretion process, going from a configuration of a stable major funnel flow to a more unstable accretion regime where several accretion tongues develop [31]. In 2011, surprisingly, AA Tau became 2 magnitudes fainter in V and the 8.2-day period disappeared from the photometry, without any variation in the mass accretion rate [11]. A possible explanation is the sudden increase of circumstellar dust extinction on the line of sight due to a nonaxisymmetric overdense region in the disk located at more than 7.7 AU that was recently brought on our line of sight.

This is the best studied CTTS in the literature, but until recently we did not know how unique was AA Tau. Is an inner disk warp a common feature in CTTSs ? Is the dynamical star-disk interaction with a timescale of a few rotational periods the rule in such systems ? Do all the inner disk warps change significantly in a timescale of years? These are questions we can start to answer with two observing campaigns of the young stellar cluster NGC 2264 that will be presented in the next section. 


\section{NGC 2264}

The young ( 2-3 Myr) stellar cluster NGC 2264, located at about $760 \mathrm{pc}$ from the Sun (see [15] for a review), was the target of two multiwavelength observing campaigns, in March 2008 and December 2011. In the first campaign it was observed for 23 days uninterruptedly in the optical by the CoRoT satellite with a simultaneous short run $(60 \mathrm{ks})$ of Chandra observations in X-rays. A few simultaneous spectroscopic and photometric observations were also obtained from the ground. In 2011 a major observing campaign was organized, called Coordinated Synoptic Investigation of NGC 2264 (CSI 2264) [13], which included 40 days of CoRoT and MOST optical photometry, 30 days of Spitzer photometry at 3.6 and $4.5 \mu \mathrm{m}$ and 300ks of Chandra/ACIS data. From the ground we obtained, spread in 3 months around the satellite observations, about 20 epochs of VLT/FLAMES multi-object spectroscopy and photometric monitoring, which included $u$ band observations with MEGACAM at CFHT.

The CoRoT light curves of CTTSs were initially classified as periodic or aperiodic. The periodic light curves include spot-like ones, due to stable spots at the stellar surface in the timescale of our observations (Fig. 3, middle), and AA Tau-like ones, due to obscuration of the stellar surface by an inner disk warp (Fig. 3, top). The aperiodic light curves were often quite complex, making it hard to attribute the observed variability to a single physical phenomenon. A few of them could however be associated to bursts of stochastic accretion (Fig. 3, bottom) and some to obscuration by circumstellar disk material.

Although the CoRoT light curves were classified based only on their morphology, in a $u-g$ vs. $g-r$ color-color diagram, the spot-like systems present in general almost no UV excess, the AA Tau-like ones present an intermediate UV excess and the burst ones show a very strong UV excess. The light curve morphology is also related to the amount of dust in the inner disk. The percentage of spot-like systems deacreses as the amount of dust in the inner disks increases. All the AA Taulike systems present inner dusty disks and the aperiodic systems dominate among the thick disks and flat spectrum sources. The CoRot light curve morphology is therefore related to the evolution of the accretion process and the inner disk structure of CTTSs.

About 20\% of the CTTS presented AA Tau-like CoRoT light curves with a flat-topped maximum interrupted by minima that varied in width and depth from cycle to cycle. They all have dust in the inner disk and, taking into account that only in high inclination systems we would be able to see the eclipses of the star by an inner disk warp, this indicates that an inner disk warp is a common feature among CTTSs.

In the 2008 campaign, the variability of CTTSs in the optical and soft X-ray $(0.5-1.5 \mathrm{keV})$ bands was found to correlate [22]. No correlation was apparent in the hard X-ray (1.5-8.0 keV) band for CTTSs, or in either bands for weak T Tauri stars, that show no sign of accretion. The observed correlation can be explained in terms of variable absorption from circumstellar material, in a scenario similar to the one proposed for AA Tau. The X-ray varibility is due to absorption of X-rays emitted in the shock region by gas in the main accretion column, while the optical variations are due to obscuration of the photosphere by dust in the inner disk warp, at the base of the main accretion column.

A good example of an AA Tau-like system is V422 Mon. In Fig. 2 (top-right), we show its light curve folded in phase, where we can see the flat-topped maximum interrupted by minima that vary in width and depth from cycle to cycle. We also show typical $\mathrm{H} \alpha$ emission line profiles outside and inside the eclipses, with clear redshifted absorption when the accretion funnel is facing the observer near phase 0.5 (Fig. 2 middle-right). The veiling is variable, increasing near the photometric minimum, when the hot spot at the base of the accretion column is passing in front of the observer (Fig. 2 bottom- 

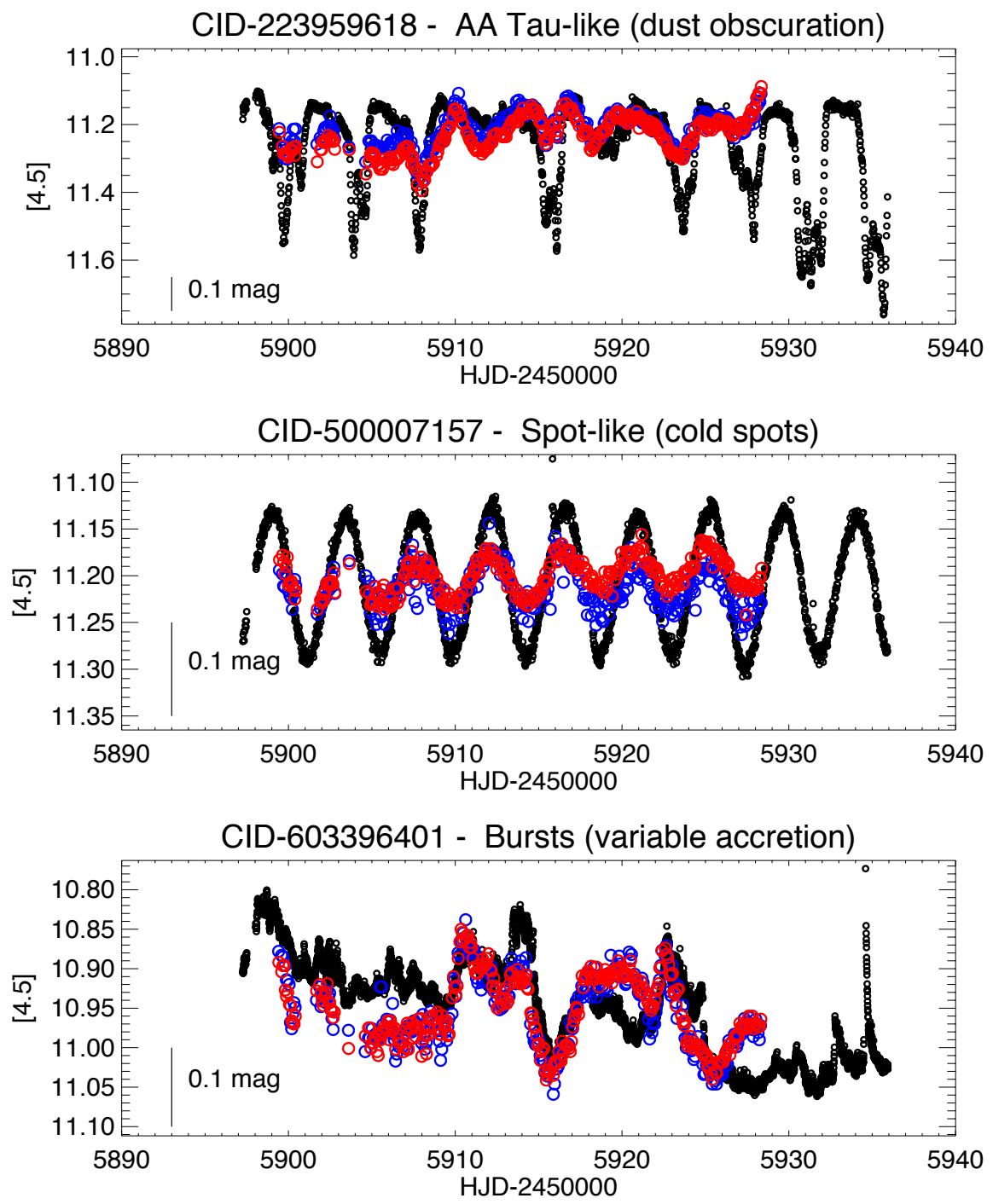

Figure 3. Examples of light curves of CTTSs observed in the CSI2264 campaign with CoRoT (black circles) in the optical and Spitzer at $3.6 \mu \mathrm{m}$ (blue) and at $4.5 \mu \mathrm{m}$ (red). The vertical axis refer to the $4.5 \mu \mathrm{m}$ magnitudes and the CoRoT and the $3.6 \mu \mathrm{m}$ light curves have been shifted by a fixed value for display purposes. Top: an AA Tau-like light curve. Middle: a spot-like light curve. Bottom: a burst-like light curve.

right). These features are nearly identical to the ones observed in AA Tau (Fig. 2, left), showing the similarity between the two systems.

In order to determine the location of the inner disk warp in AA Tau-like systems, we compared the period distribution of spot-like systems and AA Tau-like ones. In the former, the measured periods are the stellar rotation periods, $\mathrm{s}$ as spots are located at the stellar surface. In the later, the measured periods correspond to the characteritic periods of the region where the warp is located. The two period 
distributions turned out not to be identical, but they are quite similar, indicating that the obscuring material cannot be too far away from the co-rotation radius, as also seen in AA Tau [1].

One interesting feature that emerged in the second observing campaign of NGC 2264 is that about half the systems classified as AA Tau-like and observed in 2008 and 2011 presented light curves that varied from AA Tau-like to aperiodic and vice-versa. This indicates that the inner disk warp, although a common feature, may be quite variable in a timescale of years. This could also point to a typical timescale of variability between a stable accretion regime, where a major funnel flow and disk warp develop, and an unstable regime where several accretion tongues reach the star and accretion occurs at no preferential phase.

\section{Conclusions}

We have discussed the observations available for two well-studied classical T Tauri stars, V2129 Oph and AA Tau. These systems offer clues on the surface magnetic field structure and evolution. AA Tau, a highly convective CTTS, presents a mostly axisymmetric and dipolar magnetic field that did not vary much between two different epochs of observations separated by a few years. V2129 Oph is slightly more massive and has already developed a radiative core. Its surface magnetic field is more complex, presenting an octupolar component stronger than the dipole at the stellar surface. The proposed magnetic field structure of V2129 Oph was the starting point in 3D MHD simulations and the result was used to calculate Balmer line profiles, at different rotational phases, that bear a good resemblance with the observed ones. This is a good indication that the proposed magnetic field structure is indeed close to reality.

The AA Tau system is viewed at high inclination and this allow a direct view of the inner disk region, which is distorted due to its interaction with an inclined stellar magnetosphere. An inner disk warp develops that eclipses the star as the system rotates. The observed light curve presents a well defined maximum interrupted by minima that may vary in width and depth from one rotational cycle to the other. In a timescale of years, the occulting screen can show large variations. Both results indicate that the star disk interaction is dynamic in timescales of weeks and years. This is probably the best studied CTTS in the literature and it allowed to test predictions of MHD simulations such as magnetic field line inflation due to differential rotation between the magnetosphe and the inner disk region. Until recently, however, we did not know if the observed characteristics of AA Tau were typical of young accreting systems.

CTTSs have periods that typically go from 4 to 8 days and it is therefore very telescope-time consuming to follow a CTTS over several rotational cycles. Accretion and star-disk interaction are highly time variable and to establish a relation among the different observable diagnostics that go from the IR to X-rays, it is important to obtain simultaneous observations at many wavelengths. These are the reasons why until recently only a few CTTSs were studied in great detail with simultaneous observations with different techniques and at various wavelengths. In 2008 and specially in 2011 a multiwavelength campaign was organized to observe the young stellar cluster NGC 2264 with several satellites (CoRoT, Spitzer, MOST, Chandra) and ground-based facilities (VLT/FLAMES and CFHT/Megacam among others) simultaneously. This campaign allowed us to verify that AA Tau-like systems, presenting an inner disk warp and a dynamical star-disk interaction that varies in a few rotational cycles, are common among CTTSs. We also found that the inner disk warp, although a common feature among CTTSs, can vary significantly in a timescale of years.

Despite all the challenges involved in long multiwavelength observational campaigns of young stellar systems, the results are very rewarding and this is certainly the best way to look at such highly time variable and physically complex systems. 


\section{References}

[1] Alencar, S. H. P., Teixeira, P. S., Guimarães, M. M., et al., Astronomy \& Astrophysics519, A88 (2010)

[2] Alencar, S. H. P., Bouvier, J., Walter, F. M., et al., Astronomy \& Astrophysics541, A116 (2012)

[3] Argiroffi, C., Flaccomio, E., Bouvier, J., et al., Astronomy \& Astrophysics530, A1 (2011)

[4] Beck, T. L., \& Aspin, C. 2012, Astronomical Journal143, 55 (2012)

[5] Basri, G., \& Batalha, C., Astrophysical Journal363, 654 (1990)

[6] Beristain, G., Edwards, S., \& Kwan, J., Astrophysical Journal551, 1037 (2001)

[7] Bouvier, J., Covino, E., Kovo, O., Martin, E. L., Matthews, J. M., Terranegra, L., \& Beck, S. C., Astronomy \& Astrophysics299, 89 (1995)

[8] Bouvier, J., Chelli, A., Allain, S., et al., Astronomy \& Astrophysics349, 619 (1999)

[9] Bouvier, J., et al., Astronomy \& Astrophysics409, 169 (2003)

[10] Bouvier, J., et al., Astronomy \& Astrophysics463, 1017 (2007)

[11] Bouvier, J., Grankin, K., Ellerbroek, L., Bouy, H., \& Barrado, D., arXiv:1304.1487 (2013)

[12] Camenzind, M., Reviews of Modern Astronomy 3, 234 (1990)

[13] Cody, A. M., Stauffer, J. R., Micela, G., Baglin, A., \& CSI 2264 Team, Astronomische Nachrichten 334, 63 (2013)

[14] Cox, A. W., Grady, C. A., Hammel, H. B., et al., Astrophysical Journal762, 40 (2013)

[15] Dahm, S. E., Handbook of Star Forming Regions, Volume I (ASP Monograph Publications, 2008) 966

[16] Donati, J.-F., Jardine, M. M., Gregory, S. G., et al., Monthly Notices of the Royal Astronomical Society380, 1297 (2007)

[17] Donati, J.-F., Skelly, M. B., Bouvier, J., et al., Monthly Notices of the Royal Astronomical Society409, 1347 (2010)

[18] Donati, J.-F., Bouvier, J., Walter, F. M., et al., Monthly Notices of the Royal Astronomical Society412, 2454 (2011)

[19] Donati, J.-F., Gregory, S. G., Alencar, S. H. P., et al., Monthly Notices of the Royal Astronomical Society425, 2948 (2012)

[20] Edwards, S., Hartigan, P., Ghandour, L., \& Andrulis, Astronomical Journal108, 1056 (1994)

[21] Edwards, S., Fischer, W., Kwan, J., Hillenbrand, L., \& Dupree, A. K., Astrophysical Journal Letters599, L41 (2003)

[22] Flaccomio, E., Micela, G., Favata, F., \& Alencar, S. P. H., Astronomy \& Astrophysics516, L8 (2010)

[23] Goodson, A. P. \& Winglee, R. M., Astrophysical Journal524, 159 (1999)

[24] Grosso, N., Bouvier, J., Montmerle, T., et al., Astronomy \& Astrophysics475, 607 (2007)

[25] Gullbring, E., Astronomy \& Astrophysics287, 131 (1994)

[26] Gullbring, E., Calvet, N., Muzerolle, J., Hartmann, L., Astrophysical Journal544, 927 (2000)

[27] Hartigan, P., Edwards, S., \& Ghandour, L., Astrophysical Journal452, 736 (1995)

[28] Hartmann, L., Hewett, R., \& Calvet, N., Astrophysical Journal426, 669 (1994)

[29] Johns-Krull, C. M., Astrophysical Journal664, 975 (2007)

[30] Koenigl, A., Astrophysical Journal Letters370, L39 (1991)

[31] Kulkarni, A. K., \& Romanova, M. M., Monthly Notices of the Royal Astronomical Society386, 673 (2008) 
[32] Kurosawa, R., Romanova, M. M., \& Harries, T. J., Monthly Notices of the Royal Astronomical Society416, 2623 (2011)

[33] Lima, G. H. R. A., Alencar, S. H. P., Calvet, N., Hartmann, L., \& Muzerolle, J., Astronomy \& Astrophysics522, A104 (2010)

[34] Matt, S., \& Pudritz, R. E., Monthly Notices of the Royal Astronomical Society356, 167 (2005)

[35] Ménard, F., Bouvier, J., Dougados, C., Mel'nikov, S. Y., \& Grankin, K. N., Astronomy \& Astrophysics409, 163 (2003)

[36] Muzerolle J., Hartmann L., \& Calvet N., Astrophysical Journal550, 944 (2001)

[37] Romanova, M. M., Ustyugova, G. V., Koldoba, A. V., Wick, J. V., \& Lovelace, R. V. E., Astrophysical Journal595, 1009 (2003)

[38] Romanova, M. M., Ustyugova, G. V., Koldoba, A. V., \& Lovelace, R. V. E., Astrophysical Journal Letters616, L151 (2004)

[39] Romanova, M. M., Long, M., Lamb, F. K., Kulkarni, A. K., \& Donati, J.-F., Monthly Notices of the Royal Astronomical Society411, 915 (2011)

[40] Sacco, G. G., Argiroffi, C., Orlando, S., et al., Astronomy \& Astrophysics491, L17 (2008)

[41] Shu F., Najita J., Ostriker E., et al. Astrophysical Journal429, 781 (1994)

[42] Stauffer, J., et al. Astrophysical Journalsubmitted (2013)

[43] Valenti, J. A. \& Johns-Krull, C. M., Astrophysics \& Space Science292, 619 (2004)

[44] Reipurth, B., \& Aspin, C., Astrophysical Journal Letters606, L119 (2004)

[45] von Rekowski, B., \& Brandenburg, A., Astronomy \& Astrophysics420, 17 (2004)

[46] Zanni, C., \& Ferreira, J., Astronomy \& Astrophysics550, A99 (2013) 